\title{
Infrared-laser-assisted photoionization of helium by coherent extreme ultraviolet light
}

\author{
Xiao-Min Tong ${ }^{1,2, *}$ and Nobuyuki Toshima ${ }^{1}$ \\ ${ }^{1}$ Institute of Materials Science, Graduate School of Pure and Applied Sciences, University of Tsukuba, 1-1-1 Tennodai, \\ Tsukuba, Ibaraki 305-8573, Japan \\ ${ }^{2}$ Center for Computational Sciences, University of Tsukuba, 1-1-1 Tennodai, Tsukuba, Ibaraki 305-8577, Japan
}

(Received 3 March 2010; published 29 April 2010)

\begin{abstract}
We investigate the infrared (IR)-laser-assisted photoionization of He by a coherent extreme ultraviolet (euv) light solving the time-dependent Schrödinger equation. A combined field of the 13th and 15th harmonics created from the same IR laser source is used to ionize He atoms coherently. We show that the ionization probabilities oscillate as a function of the time delay between the IR and the euv pulses. On the other hand, the oscillation amplitude increases as the IR intensity increases, reaches a maximum when the IR intensity is around $6 \times 10^{12} \mathrm{~W} / \mathrm{cm}^{2}$, and then decreases as the IR intensity increases further. Decomposing the ionization probabilities by the 13th and 15th harmonics, we illustrate that the oscillation amplitude is small for a lower IR laser intensity due to the fact that the transition strength by the 13th harmonic is much smaller than the one by the 15 th harmonic. When the IR intensity increases further above $6 \times 10^{12} \mathrm{~W} / \mathrm{cm}^{2}$, the transition strength by the 13th harmonic becomes larger than the one by the 15th harmonic and the oscillation amplitude is reduced again. By tuning the relative field strengths of the 13th and 15th harmonics or the IR intensity, we can control the oscillation amplitude.
\end{abstract}

DOI: 10.1103/PhysRevA.81.043429

PACS number(s): 32.80.Qk, 32.80.Rm, 42.65.Ky

\section{INTRODUCTION}

Interference is one of the most fundamental physical processes in quantum mechanics. In the laser-atom experiments, various self-interference mechanisms of a single particle can be explored. For example, in the recent experiments [1,2], an interference which is similar to the space double-slit experiment was observed for the laser-induced electron rescattering. The modulation of the above-threshold ionization (ATI) spectra observed in the experiments [3,4] was explained as the time double-slit interference between the electrons created at different times within one laser cycle [5]. The infrared (IR)-laser-assisted He ionization yield by an attosecond pulse train (APT) also oscillates as a function of the time delay between the IR laser and the APT [6,7]. The oscillation was originally explained as the interference effect in the collision of the electron wave packets created by the different attosecond pulses (AP) in the train [8]. Recently, Tong et al. [9] proposed a general theory to explain the IR-assisted photoionization processes. In this theory, atomic structures in an IR laser field are described by Floquet states [10] and atoms can be excited or ionized to a Floquet state by a single AP (SAP) via different Floquet components. The interference of ionization channels via different Floquet components results in the oscillation of the ionization yield as a function of the arriving time of the AP. We may call this phenomenon as an energy double-slit interference since it comes from the transitions through different intermediate Fourier components. The theory explains the oscillations of the IR-assisted atomic ionization yield by the SAP or APT. Furthermore, Johnsson et al. [6] found that the oscillation amplitude also varies anomalously as a function of the IR intensity. Now the question is how to explain the anomalous dependence of the oscillation amplitude on the IR intensity.

\footnotetext{
*tong.xiaomin.ga@u.tsukuba.ac.jp
}

To investigate the physical origin of this behavior, we simplify the process as the IR-assisted photoionization of $\mathrm{He}$ by a combined field of the 13th and 15th harmonics created from the same IR laser source, which are the major components in the APT used in the experiments [6,7]. Decomposing the contributions of the 13th and 15th harmonics and varying the relative field strengths of the two euv light sources, we find that the transition strength by the 15 th harmonic is larger than that by the 13th harmonic when the IR intensity is low. As the IR intensity increases, the transition strength by the 13th harmonic increases faster than the one by the 15 th harmonic and becomes even larger than the latter. The oscillation amplitude reaches maximum when the two transition strengths are close to each other. This explains why the oscillation amplitude peaks near the IR intensity of $6 \times 10^{12} \mathrm{~W} / \mathrm{cm}^{2}$ where the two transition strengths are comparable. Excluding an individual atomic state in the simulation, we can identify the major contributor to the ionization process. In the present case, we find that the $3 p$ state is the major contributor in the low IR intensity and the $2 p$ state becomes more important as the IR intensity increases. By tuning the relative field strengths of the two euv light sources, we can manipulate the oscillatory structure. We describe the numerical procedure in Sec. II and present our numerical results and discussion in Sec. III, followed by a summary.

\section{THEORETICAL METHOD}

We study the IR-laser-assisted photoionization process of He by euv light solving the time-integral equation as (atomic units, $m=\hbar=e=1$, are used hereafter unless otherwise stated),

$$
\begin{aligned}
|\Psi(t)\rangle= & -i \int_{-\infty}^{t} e^{-i \int_{t^{\prime}}^{t} H\left(t^{\prime \prime}\right) d t^{\prime \prime}} V^{\mathrm{ext}}\left(t^{\prime}\right) e^{-i H_{0} t^{\prime}}\left|\Psi_{g}\right\rangle d t^{\prime} \\
& +e^{-i H_{0} t}\left|\Psi_{g}\right\rangle .
\end{aligned}
$$


Here, $\Psi_{g}$ is the ground state of helium atoms, $H_{0}=-\nabla^{2} / 2+$ $V(r)$ is the atomic Hamiltonian with the model potential $V(r)$ [11], $V^{\text {ext }}(t)$ is the interaction of the electron with external light fields, and the total Hamiltonian $H(t)=H_{0}+V^{\text {ext }}(t)$. In writing the above equation, we employ the independent electron approximation that only one active electron is taken into account and the other one is frozen to the atomic ground state. The IR field is written as

$$
E_{\mathrm{IR}}(t)=F_{\mathrm{IR}} e^{-2 \ln 2\left(t-t_{d}\right)^{2} / \tau^{2}} \cos \left[\omega\left(t-t_{d}\right)\right],
$$

where $F_{\mathrm{IR}}$ is the field strength, $\tau=45 \mathrm{fs}$ is the full width halfmaximum (FWHM) of the IR pulse, $\omega$ is the center frequency, and $t_{d}$ is the time delay between the IR pulse and the euv pulse. We choose the laser wavelength of $796 \mathrm{~nm}$ as used in the experiment [6]. The field of the euv pulse is written as

$$
E_{x}(t)=e^{-2 \ln 2 t^{2} / \tau_{x}^{2}}\left[F_{13} \cos (13 \omega t)+F_{15} \cos (15 \omega t)\right],
$$

where $F_{13}$ and $F_{15}$ are the field strengths of the 13th and 15th harmonics, $\tau_{x}=10$ fs the FWHM of the euv pulse. We assume that both the IR laser and euv light are linearly polarized along the $z$ direction, and thus we can write $V^{\text {ext }}(t)=-z\left[E_{\mathrm{IR}}(t)+E_{x}(t)\right]$. The wave function in Eq. (1) is propagated by the split-operator method in the energy representation [12]. In the method, we first diagonalize the Hamiltonian $H_{0}$ in an $L^{2}$ integrable basis set [13]. Then, we propagate the time-dependent wave function on this obtained field-free atomic basis. The low-lying states are the atomic bound states and we can identify the contribution of a given bound state to the dynamic process by including and excluding it in the simulation.

The numerical simulations are performed in a finite box. To avoid the unphysical reflection at the boundary, we separate the space into two regions within the box, the inner region and the outer region. When the time-dependent wave function in space reaches the outer region, we project the outer-region wave function into momentum space (Volkov state) and remove it from the wave function in real space. This procedure allows us to keep all the phase information for a long time propagation without the reflection from the boundary. The details of the numerical procedure can be found in our previous articles $[14,15]$. When the pulses are over, the total wave function is separated into two parts. One is the wave function in momentum space (or the outer region) which describes the ionization channels and the other is located in the inner region in real space and describes the excitation channels. The last term of Eq. (1) does not contribute to any dynamical process. To improve the numerical accuracy, we drop this part in the calculation. The wave function in momentum space provides all the information of the ATI spectra. Integrating the ATI spectrum we get the total ionization probability. The total ionization probability can also be obtained by summing up all the probabilities of the ejected electrons to the outer region each time before projecting the wave function into momentum space. The total ionization yields calculated by the two ways agree with each other within a few percent difference. The ionization probabilities presented in this work are calculated from the integration of ATI spectrum.

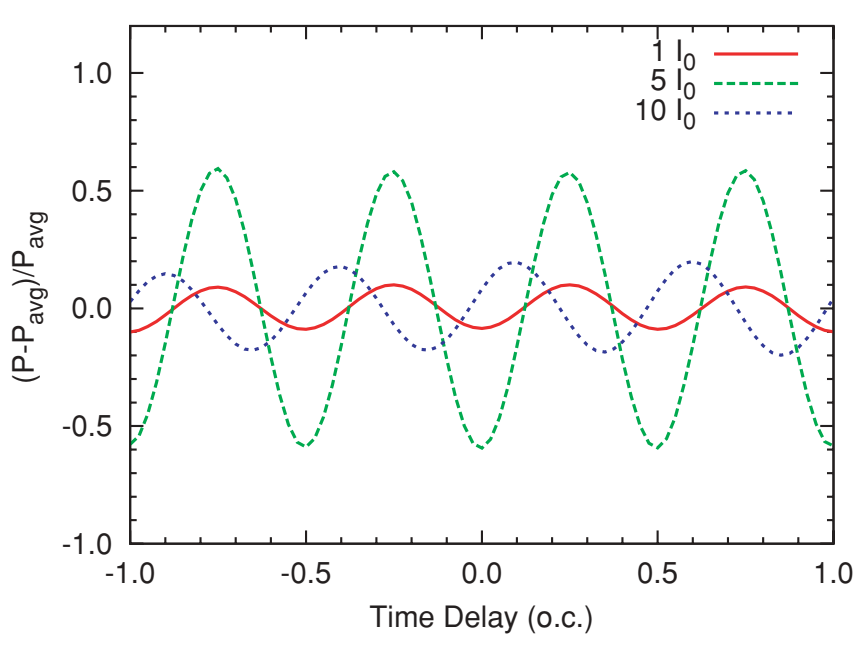

FIG. 1. (Color online) The IR-assisted photoionization probabilities of He by a combined euv light of the 13th and 15th harmonics as a function of time delays between the IR and euv pulses for different IR intensities $\left(I_{0}=10^{12} \mathrm{~W} / \mathrm{cm}^{2}\right)$; o.c. denotes optical cycle.

\section{RESULTS AND DISCUSSION}

Based on the previously described method, we study the IR-laser-assisted photoionization of $\mathrm{He}$ by the combined field of the 13th and 15th harmonics. To avoid nonlinear effect induced by the euv light, we choose a weak euv field (in the order of $10^{10} \mathrm{~W} / \mathrm{cm}^{2}$ ) and the intensity ratio of the 13th and 15 th harmonics as 1:1 hereafter unless otherwise stated. Since the euv photon energies are below the ionization threshold of $\mathrm{He}$ atoms, the euv light cannot ionize He atoms without the IR laser. The IR laser intensity used here is also so weak that the IR laser alone cannot ionize $\mathrm{He}$ atoms either. With the assistance of the IR laser, the He atoms can be ionized by the euv pulse and the ionization probability $P$ oscillates as a function of the time delay between the IR laser and euv pulses as shown in Fig. 1 . Here $P_{\text {avg }}$ is the averaged value of the ionization probability $P$ over one optical cycle for time delay $t_{d}$ from -0.5 o.c. to 0.5 o.c. (o.c. denotes optical cycle). The oscillation amplitude of the IR intensity $5 I_{0}\left(I_{0}=10^{12} \mathrm{~W} / \mathrm{cm}^{2}\right)$ is the largest among the three intensities. For $I_{0}$ and $5 I_{0}$ cases the maxima appear at the same time delays, which are different from the maxima of $10 I_{0}$ case.

Figure 2 shows the normalized amplitudes of the oscillations as a function of the IR intensity. We also plot the time delay at which the maximum ionization probability is obtained for each IR intensity. We see that the amplitude increases as the IR intensity increases and it reaches the peak near $6 I_{0}$, and then it decreases as the IR intensity increases further. The amplitude varies as a function of the IR intensity, which is consistent with Johnsson et al.'s calculation [6]. We will focus on the IR intensities from $I_{0}$ to $10 I_{0}$ to illustrate the physics of the variation.

In a recent paper [9], a general theory to understand the IR-assisted photoionization by the AP or APT was proposed. We apply this theory to explain the observed oscillations and the anomalous behavior of the maximum as a function of the IR intensity. Based on the theory, the oscillation comes from the interference of the transitions to a given Floquet state by the 


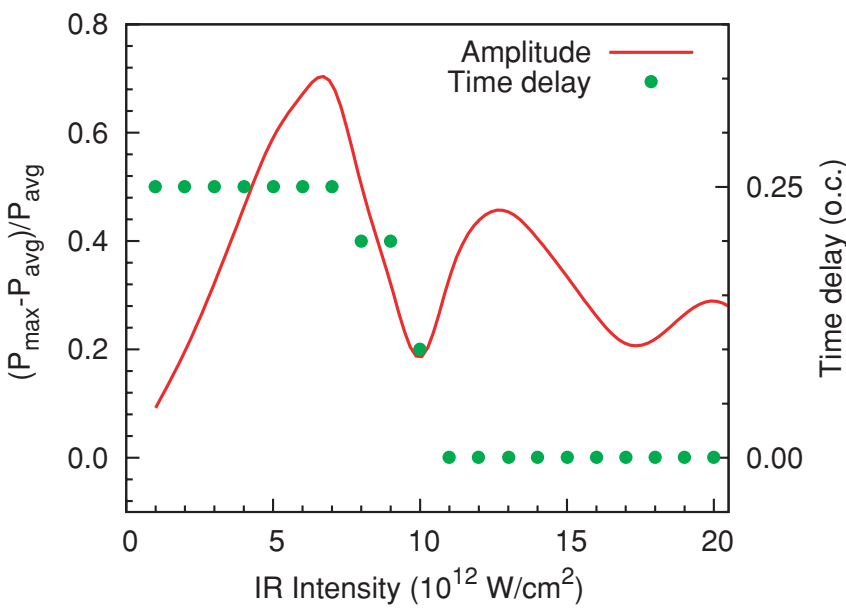

FIG. 2. (Color online) The normalized oscillation amplitudes of the IR-assisted photoionization of He by the combined euv light of the 13 th and 15 th harmonics as a function of the IR intensity. The timedelays which correspond to the maximum ionization are also plotted.

13th or 15th harmonic. The IR-assisted ionization probability by the euv light can be expressed as

$$
P\left(t_{d}\right) \propto \sum_{\alpha}\left|M_{13}^{\alpha} F_{13}+M_{15}^{\alpha} F_{15} e^{-i\left(2 \omega t_{d}\right)}\right|^{2} \delta\left(\bar{\epsilon}_{g}-\epsilon_{\alpha}\right),
$$

where $M_{13}^{\alpha}$ and $M_{15}^{\alpha}$ are the transition matrices by the 13th and 15th harmonics to a given Floquet state $\alpha, \epsilon_{\alpha}$ is the quasienergy of the Floquet state [10], and $\overline{\epsilon_{g}}=\bmod \left(\epsilon_{g}, \omega\right)$ with $\epsilon_{g}$ the ground-state energy. In writing the above equation, we factor out a common phase $13 \omega t_{d}$, which does not affect the ionization probability, to emphasize the interference between the two terms. From the equation we see that if only one high-order harmonic generation (HHG) component is used to ionized the atom, the ionization yield does not depend on the relative phase between the IR and the euv light. The transition matrix elements depend only on the IR intensity and atomic structures.

Figure 3 shows the ionization probabilities of $\mathrm{He}$ by the individual 13th or 15th harmonic, respectively, as a function of the IR intensity. The ionization probability is proportional

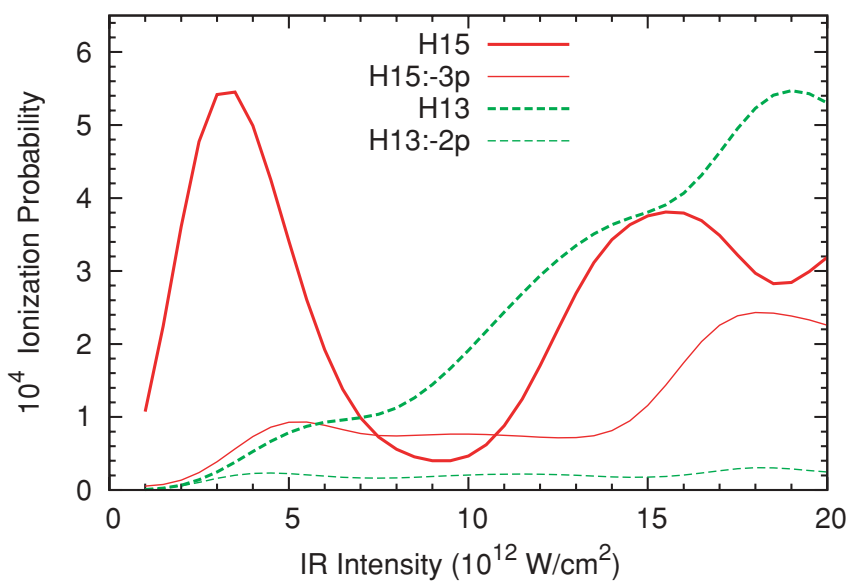

FIG. 3. (Color online) The ionization probabilities of He by the 13 th or 15 th harmonic as a function of the IR intensity. The results without the $2 p$ or $3 p$ atomic states are also presented. to the square of the transition matrix elements and independent from the relative phase between the IR laser and the euv light. The ionization probability of $\mathrm{He}$ by the 15 th harmonic takes its maximum near $3 I_{0}$ and it decreases at higher intensity. It shows a minimum near $9 I_{0}$ and then increases again. By comparing the ionization probabilities with or without the $n p$ state, we see that the $3 p$ state is the major contributor to the IR-assisted ionization by the 15 th harmonic when the intensity is lower than $6 I_{0}$. For the higher IR intensity, we cannot single out one dominant $n p$ state. For the IR-assisted ionization by the 13th harmonic at the lower IR intensity, we cannot find out one dominant $n p$ state, but we see that the $2 p$ contribution increases as the IR intensity increases and becomes dominant for higher IR intensity $\left(I>8 I_{0}\right)$. If He atoms are ionized by the 13th or 15th harmonic to the same Floquet state, the ATI spectra should be the same since the pattern of the ATI spectra for a given Floquet state does not depend on how the atom is excited to the Floquet state.

Figure 4 shows the ATI spectra of He atoms ionized by the 15th harmonic (upper panel) and the 13th harmonic (lower panel). The ATI spectra are normalized to the ionization probability. We see that in the lower IR intensity $\left(I<3 I_{0}\right)$, the two ATI spectra look similar. The first ATI peak of the 13th harmonic is broader than the one of the 15th harmonic. So we can say that there is one dominant Floquet state with some less important near-degenerate Floquet states. The ATI spectra look closer to each other in the IR intensity around $4 I_{0}$ and differ again for the higher IR intensity. The channel closing $[16,17]$ of the ATI spectra also appears at different
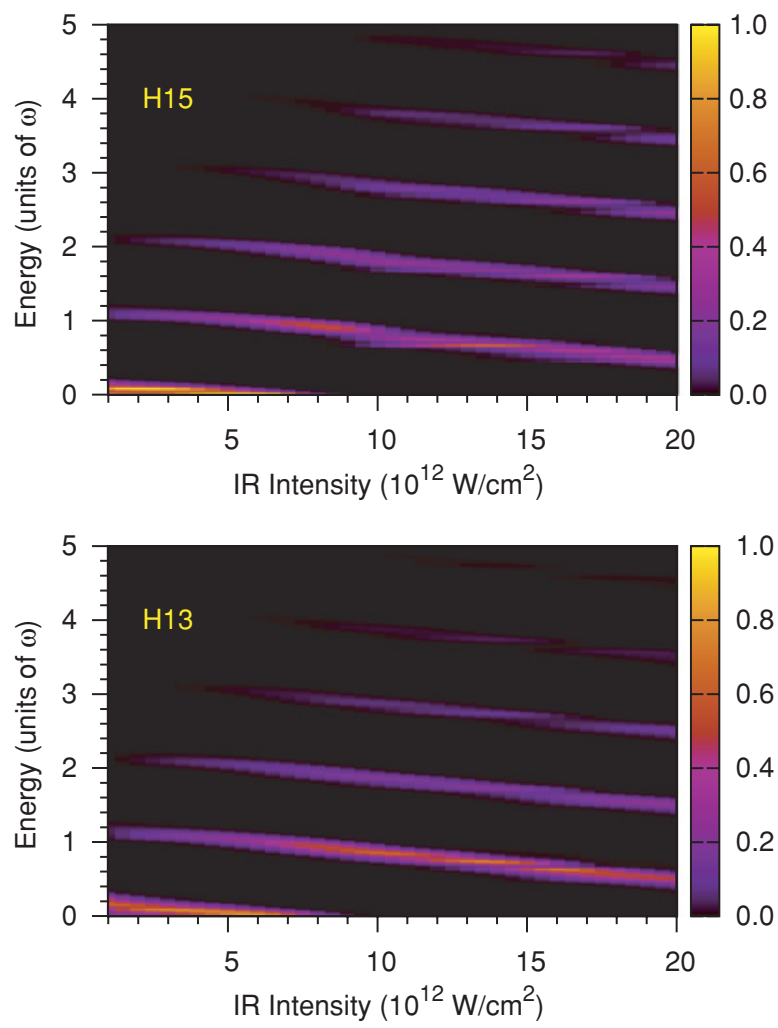

FIG. 4. (Color online) The ATI spectra of He by the15th (upper panel) or 13th (lower panel) harmonics as a function of the IR intensity. 


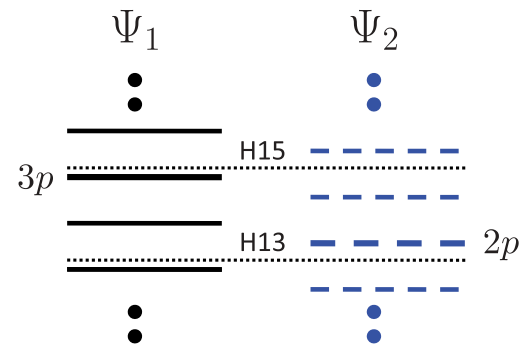

FIG. 5. (Color online) A schematic diagram of the energy structures of the two Floquet states dominated by the atomic $2 p$ and $3 p$ states. The dotted lines are the energy positions of the 13th and 15 th harmonics.

IR laser intensities for the ATI spectra of the 13th and 15th harmonics.

Let us analyze what we learned from Figs. 3 and 4. There are two dominant Floquet states contributing to the observed ATI spectra. One is mainly composed of the atomic $3 p$ state and the strong photoabsorption comes from the 15th harmonic (the major absorption Fourier component) [18] with many sidebands (including the $3 p$ state emitting two IR photons) as shown in Fig. 5. The other is mainly composed of the atomic $2 p$ state and the strong photoabsorption comes from the 13th harmonic with many sideband (including the $2 p$ state absorbing two IR photons). We call the former one as $3 p$ Floquet state and the later one as $2 p$ Floquet state as depicted in Fig. 5.

In the lower IR intensity, the photoionization is mainly through the $3 p$ Floquet state by the 15th harmonic with smaller contribution from the 13th harmonic and hence the interference by the 13th harmonic is suppressed. The 13th harmonic may also excite the $2 p$ Floquet state since there are visible high-energy components in the first ATI peak. This explains why the oscillation amplitude decreases as the IR intensity decreases. As the IR intensity increases, due to the ac stark shift [19], the transition strength to the $3 p$ Floquet state by the 15 th harmonic increases. The transition strength to the sideband of the $3 p$ state emitting two IR photons increases even faster since the coupling of the sidebands with the atomic $3 p$ state depends on the IR intensity. The ac stark shift moves the $2 p$ Floquet state far away from the resonance. Thus, the contribution of the $2 p$ Floquet state is suppressed. Comparison of the ATI spectra in Fig. 4 infers that the transitions are mainly through the $3 p$ Floquet state. This results in increase of the oscillation amplitude as the IR intensity increases. As we increase the IR intensity further, due to the ac stark shift, the transitions to both Floquet states are getting important and the two have different phase factors. Therefore, the cancellation between the two reduces the oscillation amplitude. If we look at the time delays at which the ionization probability reaches its maximum in Fig. 2, we find that the peaks appear at 0.25 o.c. time delay at low IR intensities and at 0.0 o.c. time delay at the high IR intensities. Thus, we can say that the $3 p$ Floquet state is important at the low IR intensity and the transition matrices by the 13th and 15th harmonics have a phase difference of $\pi$. The $2 p$ Floquet state is important at the high IR intensity and the transition matrices by the 13th and 15th harmonics have a phase difference of 0 . The two
Floquet states compete with each other in the intensity around $10 I_{0}$.

To test the above scenarios, we vary the pulse duration of the euv light and find that the ionization probability decreases for a longer euv light at low intensity $I_{0}$. This is because the longer pulse results in a narrow energy distribution and the energy components matching with the resonance energy are reduced. At the near-resonant intensity $\left(4 I_{0}\right)$, the ionization increases as the euv pulse duration increases.

If one Floquet state is dominant in the process, we are able to control the oscillation amplitudes by tuning the relative strengths of the 13th and 15th harmonics. For a given Floquet state, the maximum oscillation will appear when the transition amplitudes $\left[M_{13}^{\alpha} F_{13}\right.$ and $M_{15}^{\alpha} F_{15}$ in Eq. (4)] to different Fourier components are comparable to each other. In Fig. 3, we see that the transition amplitude by the 13th harmonic is much smaller than the one by the 15 th harmonic for IR intensity $I_{0}$. If we increase the intensity of the 13th harmonic to make its transition amplitude of the same order as the one by the 15 th harmonic, the oscillation amplitude increases dramatically as shown in Fig. 6. If there is only one Floquet state and the transition amplitudes to different Fourier components are the same, the oscillation amplitude $\left[\left(P-P_{\text {avg }}\right) / P_{\text {avg }}\right]$ should reach unity. This implies that there must be more than one Floquet state that contributes to the ionization.

The ATI spectra by the 13th and 15th harmonics are close to each other at $4 I_{0}$ in Fig. 4, this implies that we may ionize helium through a common Floquet state by the 13th and 15th harmonics. If we tune the intensity of the 13th harmonic to make the transition amplitude close to the one by the 15 th harmonic, we can get a larger oscillation as shown in Fig. 7. The increase of the oscillation amplitude in Fig. 7 does not look so dramatic since the ionization probability is normalized to the averaged value. In fact, the ratio of the maximum to the minimum ionization probabilities increases from 2.7 to 8.3. By comparing the spectra with and without the atomic $3 p$ state, we conclude that the oscillation comes from a Floquet state

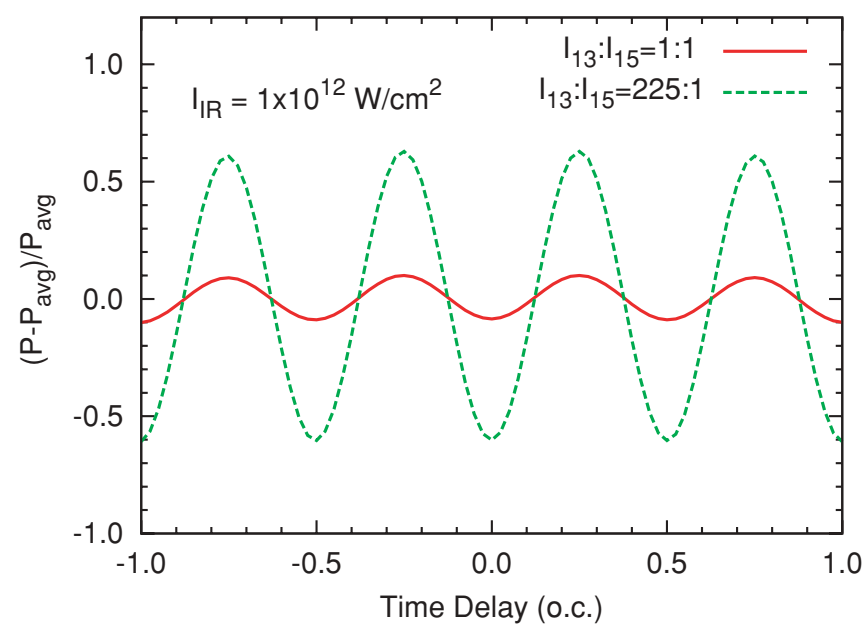

FIG. 6. (Color online) The photoionization probabilities of He by a combined euv light of the 13th or 15th harmonics as a function of the time delay between the IR and XUV pulses by varying the relative field strengths of the 13 th and 15 th harmonics for $I_{\mathrm{IR}}=$ $1 \times 10^{12} \mathrm{~W} / \mathrm{cm}^{2}$. 


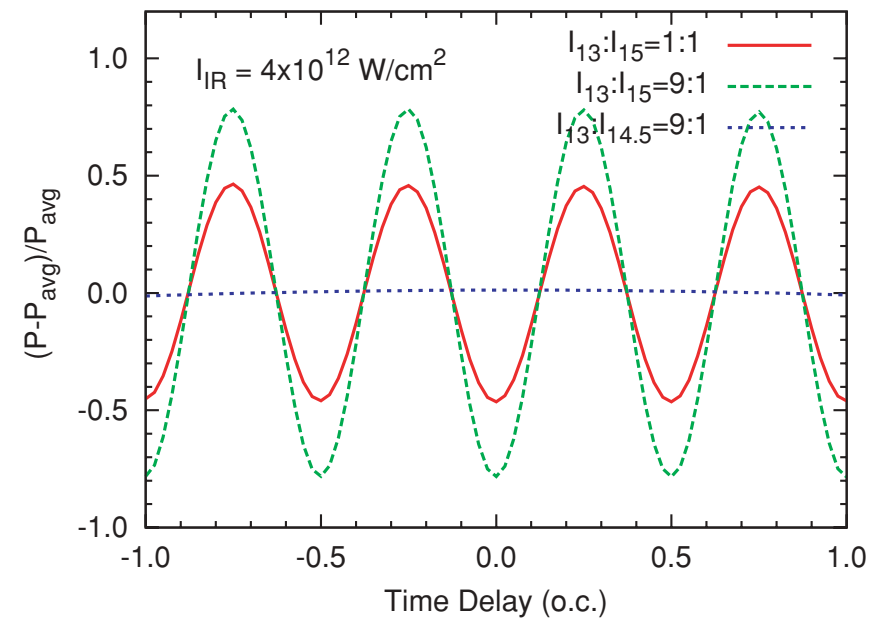

FIG. 7. (Color online) The same as Fig. 6 but for $I_{\text {IR }}=4 \times$ $10^{12} \mathrm{~W} / \mathrm{cm}^{2}$.

relevant to the $3 p$ state. To confirm that the oscillation is due to the transitions to different Fourier components of a given Floquet state, we artificially shift the 15th harmonic energy to $14.5 \omega$ so that the ionization by the two euv light sources cannot go through a same Floquet state. With this manipulation, the oscillation completely disappears as shown in Fig. 7.

If two or more Floquet states are involved in the competition region, the oscillation amplitudes are not very sensitive to the intensities of the 13th and 15th harmonics and the oscillation amplitude cannot be enhanced significantly as shown in Fig. 8. We suppose that there are at least two dominant Floquet states contributing to the ATI spectra when the IR intensity is $10 I_{0}$. If we increase the field strength of the 15 th harmonic, both transition strengths increase but the oscillation amplitude does not change much. If we increase the field strength of the 13th harmonic, the transition strength to the $2 p$ Floquet state increases which makes the total oscillation amplitude decrease. As we increase the IR intensity further, more and more Floquet states are involved and the oscillation pattern is getting more complicated but the physical processes are the same.

To summarize, using the recent proposed mechanism of the IR-assisted photoabsorption of atoms by AP or APT, we explained why the oscillation amplitude changes anomalously as the IR intensity changes. At the low IR intensity, the oscillation amplitude is reduced due to the fact that the transition matrix element by the 15th harmonic is much larger than the one by the 13th harmonic euv light. Tuning the relative field strengths of the 13 th and 15 th harmonics, we can

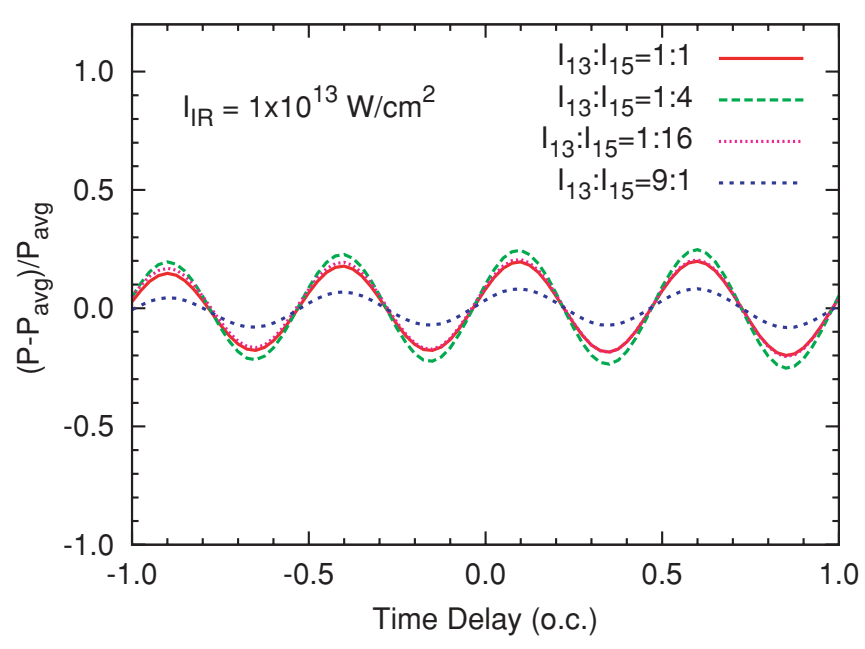

FIG. 8. (Color online) The same as Fig. 5 but for $I_{\mathrm{IR}}=1 \times$ $10^{13} \mathrm{~W} / \mathrm{cm}^{2}$.

enhance the oscillation significantly for this case. At the high IR intensity near $10^{13} \mathrm{~W} / \mathrm{cm}^{2}$, the oscillation is suppressed due to the competition of two equally important Floquet states with different phases. The oscillation amplitude cannot be enhanced significantly by tuning the relative field strengths of the two euv light sources for this case. The two routes through the energy double slit can be tuned either by varying the IR intensity or the relative field strengths of the two euv light sources. The control of the IR intensity tunes the atomic structure and the relative strength of the euv light sources tunes the light current going through different energy slits. The interference strength can be well controlled by these two quantities. For controlling the dynamics more effectively, it is important to know how the photoabsorption process is modified by the IR intensity as well as the bound state energy shifts by the IR laser. Once we have the systematic information about it, we will know how to control an atomic system more effectively by the IR laser and ionize it by a coherent euv light source, like AP and APT, which are already available in several laboratories [20-23].

\section{ACKNOWLEDGMENTS}

The authors thank Prof. C. L. Cocke and Dr. P. Ranitovic for their useful discussions. The numerical calculations were carried out by the T2K-Tsukuba System in the Center for Computational Sciences, University of Tsukuba. This work was supported by Grant-in-Aid for Scientific Research (C) from the Japan Society for the Promotion of Science.
[1] D. Akoury et al., Science 318, 949 (2007).

[2] M. Okunishi, R. Itaya, K. Shimada, G. Prümper, K. Ueda, M. Busuladžić, A. Gazibegović-Busuladžić, D. B. Milošević, and W. Becker, Phys. Rev. Lett. 103, 043001 (2009).

[3] F. Lindner, M. G. Schätzel, H. Walther, A. Baltuška, E. Goulielmakis, F. Krausz, D. B. Milošević, D. Bauer, W. Becker, and G. G. Paulus, Phys. Rev. Lett. 95, 040401 (2005).

[4] R. Gopal et al., Phys. Rev. Lett. 103, 053001 (2009).

[5] D. G. Arbó, E. Persson, and J. Burgdörfer, Phys. Rev. A 74, 063407 (2006).
[6] P. Johnsson, J. Mauritsson, T. Remetter, A. L’Huillier, and K. J. Schafer, Phys. Rev. Lett. 99, 233001 (2007).

[7] P. Ranitovic et al., New J. Phys. 12, 013008 (2010).

[8] P. Riviere, O. Uhden, U. Saalmann, and J. M. Rost, New J. Phys. 11, 053011 (2009).

[9] X. M. Tong, P. Ronitovic, C. L. Cocke, and N. Toshima, Phys. Rev. A 81, 021404(R) (2010).

[10] S. I. Chu and D. A. Telnov, Phys. Rep. 390, 1 (2004).

[11] X. M. Tong and C. D. Lin, J. Phys. B 38, 2593 (2005).

[12] X. M. Tong and S. I. Chu, Chem. Phys. 217, 119 (1997). 
[13] D. A. Telnov and S. I. Chu, Phys. Rev. A 59, 2864 (1999).

[14] X. M. Tong, K. Hino, and N. Toshima, Phys. Rev. A 74, 031405(R) (2006).

[15] X. M. Tong, S. Watahiki, K. Hino, and N. Toshima, Phys. Rev. Lett. 99, 093001 (2007)

[16] G. G. Paulus, F. Grasbon, H. Walther, R. Kopold, and W. Becker, Phys. Rev. A 64, 021401(R) (2001).

[17] B. Borca, M. V. Frolov, N. L. Manakov, and A. F. Starace1, Phys. Rev. Lett. 88, 193001 (2002).
[18] T. Shirahama, X.-M. Tong, K.-I. Hino, and N. Toshima, Phys. Rev. A 80, 043414 (2009).

[19] S.-I. Chu and J. Cooper, Phys. Rev. A 32, 2769 (1985).

[20] M. Drescher, M. Hentschel, R. Kienberger, G. Tempea, C. Spielmann, G. A. Reider, P. B. Corkum, and F. Krausz, Science 291, 1923 (2001).

[21] G. Sansone et al., Science 314, 443 (2006).

[22] E. Goulielmakis et al., Science 320, 1614 (2008).

[23] I. Thomann, A. Bahabad, X. Liu, R. Trebino, M. M. Murnane, and H. C. Kapteyn, Opt. Express 17, 4611 (2009). 